\title{
Enfoques de aprendizaje y rendimiento académico en estudiantes de medicina del primer año de la Universidad Nacional Mayor de San
} Marcos

\section{Learning approaches and academic performance of first year medical students of National Major University of San Marcos}

\author{
Heli Jaime Barrón Pastor ${ }^{1, a, b}$, Yesica Llimpe Mitma de Barrón ${ }^{1,2, a}$ \\ ${ }^{1}$ Departamento Académico de Ciencias Dinámicas, Facultad de Medicina, Universidad Nacional Mayor de San Marcos, Lima, Perú. \\ ${ }^{2}$ Instituto Nacional de Enfermedades Neoplásicas, Lima, Perú. \\ ${ }^{a}$ Magister en Bioquimica; ${ }^{b}$ Associate Fellow of Higher Education Academy (HEA), York, United Kingdom; Master in Health Professions Education, Maastricht University, The \\ Netherlands.
}

\section{Correspondencia}

Heli Jaime Barrón Pastor helibarron@unmsm.edu.pe Departamento Académico de Ciencias Dinámicas, Facultad de Medicina, Universidad Nacional Mayor de San Marcos. Av. Grau 755, Lima 1, Perú.

Recibido, 20 abril 2016

Evaluado, 1 agosto 2016

Aceptado, 29 agosto 2016

Fuente de financiamiento: personal Ningún conflicto de interes.

Citar como: Barrón Pastor HJ, Llimpe Mitma de Barrón Y. Enfoques de aprendizaje y rendimiento académico en estudiantes de medicina del primer año de la Universidad Nacional Mayor de San Marcos. An Fac med. 2017;78(1):4954. DOI: http://dx.doi.org/10.15381/ anales.v78i1.13021
An Fac med. 2017;78(1):49-54 / http://dx.doi.org/10.15381/anales.v78i1.13021

\section{Resumen}

Introducción. Cada año, miles de estudiantes de medicina se enfrentan al desafío de superar las asignaturas para adquirir las competencias para sus actividades profesionales. El éxito de la vida estudiantil en camino a la profesión depende de muchos factores incluyendo los enfoques de aprendizaje. Objetivo. Determinar los enfoques de aprendizaje predominantes de los estudiantes de medicina del primer año y su relación con el rendimiento académico. Diseño. Estudio observacional, descriptivo, de corte transversal, cuantitativo. Lugar. Facultad de Medicina, Universidad Nacional Mayor de San Marcos, Lima, Perú. Participantes. Estudiantes de la Facultad de Medicina. Intervenciones. A 130 estudiantes de la Facultad de Medicina matriculados en la asignatura de Biología celular y molecular, se aplicó una versión corta del cuestionario Approaches and Study Skills Inventory for Students (ASSIST) y se evaluó la relación de enfoques de aprendizaje con rendimiento académico. La asociación de las subescalas del cuestionario se evaluó mediante análisis factorial y la consistencia interna mediante análisis alfa de Cronbach. La relación de cada enfoque de aprendizaje y el rendimiento académico se evaluó mediante análisis de regresión. El efecto de la edad, sexo y sus interacciones con cada enfoque de aprendizaje se evaluó con la prueba ANOVA de dos vias. Principales medidas de resultados. Puntaje de los enfoques de aprendizaje profundo, estratégico y superficial. Resultados. El puntaje total de los enfoques de aprendizaje profundo, estratégico y superficial fueron 22,4, 20,4 y 15,4, respectivamente. Hubo correlación significativa entre la puntuación de exámenes teóricos y enfoque estratégico $(p=0,004)$. No se encontraron diferencias con relación a edad y sexo. Conclusiones. El enfoque profundo fue el predominante en estudiantes de medicina del primer año de la Universidad Nacional Mayor de San Marcos. Se encontró correlación significativa entre el enfoque estratégico y el rendimiento académico.

Palabras clave. Enfoques de Aprendizaje; Cuestionario ASSIST; Estudiantes de Medicina; Rendimiento Académico.

Abstract

Introduction: Every year thousands of medical students face the challenge of overcoming academic subjects to acquire skills for their professional activities. The success of the student's life on the way to the profession depends on many factors including learning approaches. Objective: To determine the predominant learning approaches in the first year medical students and their relationship to academic achievement. Design: Observational, descriptive, quantitative study. Institution: Faculty of Medicine, Universidad Nacional Mayor de San Marcos, Lima, Peru. Participants: Medical students. Interventions: The short version of the questionnaire Approaches and Study Skills Inventory for Students (ASSIST) was applied to 130 first year medical students enrolled in the Cellular and Molecular Biology subject; the relationship of learning approaches with academic performance was evaluated. The association of the subscales was assessed by factorial analysis and the internal consistency by Cronbach's alpha analysis. The relationship of each approach to learning and academic performance was evaluated by regression analysis. The effect of age, gender and their interactions with each learning approach were evaluated by a two-way ANOVA. Main outcome measures: Deep, strategic and superficial learning approaches score. Results: The total score for deep, strategic and superficial learning approaches were $22.4,20.4$, and 15.4 respectively. There was significant correlation between theoretical examination scores and strategic approach $(p=0.004)$. No differences in relation to age and sex were found. Conclusions: The deep approach was predominant in San Marcos first year medical students. There was high significant correlation between the theoretical examinations and the strategic approach.

Keywords: Learning Approaches; ASSIST Inventory; Medical students; Academic Performance. 


\section{INTRODUCCIÓN}

Cada año miles de estudiantes se enfrentan al desafío de superar las asignaturas académicas para adquirir las competencias necesarias para el desarrollo de sus actividades profesionales. De acuerdo con la teorías de aprendizaje, el éxito de la vida estudiantil en camino a la profesión depende de muchos factores, incluyendo la influencia de factores educativos como socioculturales para el aprendizaje y la motivación ${ }^{(1)}$. La mayoría de los estudios sobre la teoría del aprendizaje resaltan la importancia central de la interacción aprendizaje - enseñanza. Existe una creciente atención en la autonomía de los estudiantes, y la función del maestro (docente) ha cambiado. Lo que el estudiante hace es más importante que lo que hace el profesor ${ }^{(2)}$. De hecho, en el proceso aprendizaje-enseñanza se habla de estudiante aprendizaje en lugar de profesor y enseñanza ${ }^{(2,3)}$. La idea principal que se desprende de estos estudios es que los estudiantes pueden tener diferentes enfoques de aprendizaje. Para el aprendizaje y el estudio, los estudiantes utilizan tres enfoques: profundo, superficial y estratégico. El uso de estos enfoques varía según las circunstancias y no son mutuamente excluyentes $(4,5)$. Factores como la edad, sexo y condición socioeconómica se han relacionado con los enfoques de aprendizaje ${ }^{(6)}$.

Los enfoques de aprendizaje son un reflejo de los diferentes niveles de procesamiento de información y no solo está referido a las diferencias individuales de propósitos y motivos cuando los estudiantes se enfrentan a una situación de aprendizaje, sino que también están relacionados con la utilización de las estrategias correspondientes ${ }^{(7)}$. En el enfoque profundo, los estudiantes tienen motivación intrínseca para entender las ideas por sí mismos. En el enfoque superficial la motivación es extrínseca, esto es aprendizaje por memorización y ausencia de propósitos. En tanto que, el estudiante que emplea el enfoque estratégico tiene la motivación de obtener las calificaciones más altas posibles usando estrategias de comprensión y memorización ${ }^{(7,8)}$.

Varios estudios han evaluado diferentes aspectos de los enfoques de apren- dizaje en ingeniería, ciencias sociales y educación médica (7,9-11). Sin embargo, el enfoque de aprendizaje en las ciencias básicas en los estudiantes de medicina de primer año ha recibido poca atención, especialmente en los aspectos relacionados con el éxito académico, y la mayoría de los hallazgos son objeto de controversia. Los ejemplos incluyen estudios realizados en Europa y Estados Unidos, tanto en ciencias aplicadas como clínicas, para vincular los enfoques de aprendizaje y el rendimiento académico ${ }^{(8,12)}$ y para evaluar las diferencias entre los enfoques de aprendizaje en estudiantes de medicina de pregrado y posgrado ${ }^{(13)}$. Byrne y colaboradores, en un estudio realizado en Irlanda encontraron que el enfoque profundo $y$ estratégico se asocia positivamente con un alto rendimiento académico; sin embargo, esta relación fue evidente solo para estudiantes de sexo femenino ${ }^{(4)}$. Por otro lado, un estudio publicado en 2010 por Diset y colaboradores, llevado a cabo para validar el cuestionario ASSIST en un país escandinavo, ha puesto de manifiesto que, como era de esperar, existe una correlación negativa entre el enfoque profundo y superficial. Sin embargo, ambos enfoques pueden correlacionarse ya sea positiva o negativamente con el enfoque estratégico, lo que sugiere la importancia del análisis factorial en los estudios para evaluar los enfoques de aprendizaje ${ }^{(8)}$. En un estudio reciente ${ }^{(13)}$ que comparó los enfoques de aprendizaje en los estudiantes de primer año, del último año y estudiantes de posgrado, el enfoque predominante fue el estratégico en todos los grupos, con un cambio positivo hacia enfoques estratégicos y profundos en los estudiantes de posgrado. Aunque la finalidad de los distintos planes de estudio en los estudiantes de medicina es promover el enfoque profundo ${ }^{(14)}$, ya que se relaciona mejor con los aspectos de la práctica clínica ${ }^{(15)}$, los resultados siguen siendo polémicos ${ }^{(6,13)}$. Sobre la base de estos resultados controversiales consideramos llevar a cabo una investigación en los estudiantes de medicina de primer año para evaluar los enfoques de aprendizaje y rendimiento académico en las ciencias básicas y la evaluación de sus diferencias.
El principal objetivo fue determinar cuál es el enfoque de aprendizaje predominante en estudiantes de medicina de primer año matriculados en la asignatura de Biología Celular y Molecular. También se evaluó si había alguna relación entre los enfoques de aprendizaje y el desempeño académico en ciencias básicas en los estudiantes de medicina de primer año.

\section{MÉTODOS}

El estudio tuvo un diseño observacional descriptivo. El muestreo fue no probabilístico por conveniencia, tipo censo, para lo cual se invitó a participar al total de estudiantes del primer año $(\mathrm{N}=198)$ que asistían regularmente a las sesiones de aprendizaje de la asignatura de Biología Celular y Molecular ${ }^{(16)} .130$ estudiantes decidieron participar en el estudio, de los cuales 62 fueron varones y 68 mujeres. El estudio se realizó en la Universidad Nacional Mayor de San Marcos, Lima, Perú, en el año 2014. El instrumento que se utilizó tenía dos secciones. La primera sección para recolectar datos sociodemográficos y la segunda para evaluar los enfoques de aprendizaje utilizando una versión corta del cuestionario ASSIST. EI estudio contó con la aprobación Comité de Ética de la Facultad de Medicina de la UNMSM. Los estudiantes firmaron un consentimiento informado para participar en el estudio.

Los enfoques de aprendizaje fueron evaluados con una versión corta del cuestionario ASSIST ${ }^{(17)}$. Esta versión consta de un total de 18 preguntas, 6 para cada enfoque de aprendizaje, que se responden de acuerdo a la escala de Likert de 5 puntos (1= 'en desacuerdo' a 5 = 'de acuerdo') ${ }^{(6,8,18)}$. Los datos se procesaron calculándose la media de puntuaciones de cada enfoque de aprendizaje (profundo, estratégico y superficial). En la tabla 1 se presenta las preguntas que identificaron a cada subescala.

La versión corta de ASSIST es un inventario diseñado, validado y prometedor que comprende solo 18 ítems y tres subescalas, cuyo número de ítems es mucho menor en comparación con la versión larga del mismo cuestionario que contiene 
Tabla 1. Preguntas usadas en la versión corta del cuestionario ASSIST para evaluar los enfoques de aprendizaje en estudiantes de medicina de la UNMSM.

... Responde marcando: 5= de acuerdo 4= de acuerdo en parte 2= en desacuerdo en parte 1= en desacuerdo. Trata de no responder 3 (ni de acuerdo ni en desacuerdo) a menos que realmente no sepas o si crees que el comentario no es aplicable en este curso.

\begin{tabular}{|c|c|c|c|c|c|c|}
\hline S01. & A menudo tengo problemas para dar sentido a las cosas que tengo que recordar & 1 & 2 & 3 & 4 & 5 \\
\hline D02. & Cuando leo un apunte o libro, trato de descubrir por mí misma/o lo que el autor quiere decir exactamente & 1 & 2 & 3 & 4 & 5 \\
\hline T03. & Yo organizo mi tiempo de estudio con cuidado para hacer el mejor uso de ella & 1 & 2 & 3 & 4 & 5 \\
\hline S04. & No hay mucho en este curso que me parece interesante o relevante & 1 & 2 & 3 & 4 & 5 \\
\hline T05. & Trabajo de manera constante a través de todo semestre, en lugar de dejar todo hasta el último minuto & 1 & 2 & 3 & 4 & 5 \\
\hline D06. & Antes de abordar un problema o una tarea, primero trato de averiguar qué hay detrás de él & 1 & 2 & 3 & 4 & 5 \\
\hline T07. & Soy bastante bueno en ponerme a trabajar (estudiar) siempre que necesito hacerlo & 1 & 2 & 3 & 4 & 5 \\
\hline S08. & Gran parte de lo que estoy estudiando tiene poco sentido: son como piezas no relacionadas & 1 & 2 & 3 & 4 & 5 \\
\hline T09. & Pongo mucho esfuerzo en el estudio, porque estoy decidido a hacerlo bien & 1 & 2 & 3 & 4 & 5 \\
\hline D10. & Cuando estoy trabajando en un nuevo tema, trato de imaginarme cómo encajan todas las ideas & 1 & 2 & 3 & 4 & 5 \\
\hline T11. & No me parece difícil motivarme a mí mismo & 1 & 2 & 3 & 4 & 5 \\
\hline D12. & A menudo me encuentro cuestionando cosas que oigo en conferencias o que leído en los libros & 1 & 2 & 3 & 4 & 5 \\
\hline T13. & Creo que soy muy sistemático y organizado & 1 & 2 & 3 & 4 & 5 \\
\hline S14. & Muchas veces siento que me ahogo en la enorme cantidad de material que tengo que estudiar & 1 & 2 & 3 & 4 & 5 \\
\hline D15. & Las ideas que encuentro en los libros o apuntes del curso a menudo me generan asociaciones de ideas propias & 1 & 2 & 3 & 4 & 5 \\
\hline S16. & En realidad, no estoy segura/o de qué es lo importante de las clases, así que trato de incorporar todo lo que puedo & 1 & 2 & 3 & 4 & 5 \\
\hline D17. & Cuando leo, examino cuidadosamente los detalles para ver cómo se ajustan a lo que se está diciendo & 1 & 2 & 3 & 4 & 5 \\
\hline S18. & A menudo me quedo preocupada/o si seré capaz de hacer frente a la tareas debidamente & 1 & 2 & 3 & 4 & 5 \\
\hline
\end{tabular}

52 ítems de los mismos tres enfoques de aprendizaje: profundo, superficial y estratégico.

La validación del instrumento fue realizada por traducción inversa para juzgar la equivalencia semántica, por análisis discriminante (componentes principales) para evaluar la coherencia y por análisis de consistencia interna (test de Cronbach) para evaluar confiabilidad.

El cuestionario fue traducido al idioma español con el fin de ser aplicado a la población local. Se realizó traducción inversa para evaluar la equivalencia semántica, el mismo que fue evaluado por un personal académico de la Facultad de Medicina de Maastricht University (Holanda). La versión definitiva del cuestionario se administró a todos los participantes después de un mínimo de ajustes en base a las recomendaciones.

Los datos fueron analizados mediante el paquete estadístico SPSS versión 21.

\section{RESULTADOS}

Primero se realizó el análisis factorial para evaluar la asociación de las subescalas propuestas en el cuestionario ASSIST mediante el análisis de componentes principales (ACP); esto con la finalidad de confirmar la asociación de los tres grupos de preguntas, sobre la base de los tres enfoques de aprendizaje. El método de extracción se realizó en base a ACP con el método de rotación Promax y Normalización Kaiser.

La tabla 2 proporciona los resultados obtenidos a partir del análisis de todos los elementos.

El análisis de datos reveló que las preguntas relacionadas con el enfoque profundo (preguntas con el identificador D) se agruparon en el primer factor, las relacionadas con el enfoque estratégico (preguntas con el identificador S) en el segundo factor y las relacionadas con el enfoque estratégico (identificador T) en el tercer factor del ACP. Sin embargo, algunas preguntas relacionadas a enfoque superficial (S4 y S8) tuvieron carga baja y fueron ubicados en el tercer factor.

El ACP reveló que 2 preguntas (T07 y T09) presentaban 2 cargas (en el enfoque estratégico y superficial) que solo deberían de aparecer en el enfoque estratégico, pero que aparecieron también en el enfoque profundo. Por otro lado, los mismos elementos parecían tener doble carga, lo que significa que estas preguntas podrían estar correlacionadas para ambos enfoques.

Se encontró que el enfoque predominante en los estudiantes de medicina del primer año matriculados en la asignatura de Biología Celular y Molecular durante el año 2014 fue el de tipo profundo, seguido del enfoque estratégico y superficial (tabla 3).

La consistencia interna del cuestionario evaluada mediante el coeficiente alfa de Cronbach indica que la confiabilidad 
Tabla 2. Análisis factorial (ACP) de la versión corta del cuestionario ASSIST aplicado a los estudiantes.

\begin{tabular}{cccc} 
Item & Componente 1 & Componente 2 & Componente 3 \\
D02 & 0,7 & 0,3 & $-0,4$ \\
D06 & 0,6 & 0,6 & $-0,1$ \\
D10 & 0,9 & 0,4 & $-0,3$ \\
D12 & 0,5 & 0,3 & 0,1 \\
D15 & 0,8 & 0,3 & $-0,2$ \\
D17 & 0,8 & 0,3 & $-0,2$ \\
S01 & $-0,4$ & $-0,4$ & 0,6 \\
S04 & $-0,8$ & $-0,1$ & 0,1 \\
S08 & $-0,8$ & $-0,2$ & 0,3 \\
S14 & $-0,1$ & $-0,2$ & 0,8 \\
S16 & $-0,3$ & $-0,2$ & 0,5 \\
S18 & $-0,1$ & $-0,1$ & 0,8 \\
T03 & 0,3 & 0,8 & $-0,2$ \\
T05 & 0,3 & 0,8 & $-0,3$ \\
T07 & 0,6 & 0,5 & $-0,3$ \\
T09 & 0,7 & 0,5 & $-0,3$ \\
T11 & 0,5 & 0,5 & $-0,4$ \\
T13 & 0,2 & 0,8 & $-0,1$ \\
\hline
\end{tabular}

de las subescalas del cuestionario usado en el presente estudio fue satisfactoria ${ }^{(19)}$.

Se realizó análisis de correlación entre las subescalas para evaluar si eran o no eran independientes. La correlación de los tres enfoques de aprendizaje fue significativa ( $p<0,01)$. La correlación entre el enfoque profundo y estratégico fue positiva $(r=0,5)$. La correlación entre el enfoque profundo y superficial y la correlación entre los enfoques estratégicos y de superficie fueron negativas, con valores $r-0,5$ y-0,4, respectivamente.

El $26 \%$ de los participantes en el estudio tenía menos de 18 años de edad, con una edad media de 19 y rangos de 16 a 23 años. El análisis descriptivo relacionado con el género y el tipo de la escuela secundaria de procedencia mostró que no hubo predominio poblacional basado en género; sin embargo, la población femenina $(52,3 \%)$ fue ligeramente mayor que la de varones. La mayoría de los estudiantes de medicina de primer año provenía de escuelas secundarias privadas
85 (65,4\%). Solo un alumno no había recibido una formación universitaria previa antes de entrar a la escuela de medicina. Solo $8 \%$ terminó la escuela secundaria un año antes de ser admitido en la facultad de medicina.

Uno de los puntos de la encuesta demográfica incluyó la percepción de los estudiantes sobre la calidad de la educación recibida en la escuela secundaria. Solo $12 \%$ consideró era muy buena, $32 \%$ consideró que era buena. La mayoría (39\%) consideró que era apropiada, 17 estudiantes (13\%) la consideraron mala y 5 alumnos (4\%) consideraron que la educación secundaria que recibieron era muy mala.

Tabla 4. Puntaje total de los enfoques de aprendizaje por sexo.

\begin{tabular}{cccc} 
& $\begin{array}{c}\text { Superficial } \\
\text { Media y DE }\end{array}$ & $\begin{array}{c}\text { Profundo } \\
\text { Media y DE }\end{array}$ & $\begin{array}{c}\text { Estratégico } \\
\text { Media y DE }\end{array}$ \\
Masculino & $15,4(4,7)$ & $22,4(4,7)$ & $20,7(5,2)$ \\
Femenino & $15,3(4,8)$ & $22,3(4,8)$ & $20,1(5,1)$ \\
\hline Total & $15,4(4,7)$ & $22,4(4,7)$ & $20,4(5,1)$ \\
\hline
\end{tabular}

Tabla 3. Puntuación media calculada para cada enfoque y su correspondiente análisis de consistencia interna mediante análisis alfa de Cronbach.

\begin{tabular}{lcc} 
& $\begin{array}{c}\text { Puntaje } \\
\text { promedio }\end{array}$ & $\begin{array}{c}\text { Alfa } \\
\text { Cronbach's }\end{array}$ \\
Enfoque superficial & 2,6 & 0,7 \\
Enfoque profundo & 3,7 & 0,8 \\
Enfoque estratégico & 3,4 & 0,8 \\
\hline
\end{tabular}

En cuanto al rendimiento académico, se encontró que el promedio de las evaluaciones tanto de teoría como de práctica fue de 13 , con valores máximos de 17,5 en teoría y 16,9 en práctica (figura 1); se consideró que la escala de evaluación en la facultad de medicina es tipo vigesimal de 0 a 20, con valor mínimo aprobatorio de 11 .

El análisis de correlación lineal usando el coeficiente $r$ de Pearson reveló que la puntuación media de los exámenes teóricos y prácticos se relacionó significativamente $(r=0,8 ; p<0,01)$. Las puntuaciones más altas en exámenes de teoría se asociaron con las puntuaciones más altas en los exámenes prácticos.

No existió relación entre el enfoque superficial y el rendimiento académico en los exámenes teóricos ( $r=-0,087$; $p=0,328)$. No hubo correlación significativa con el enfoque profundo $(r=0,126$; $p=0,153)$. Sin embargo, sí hubo correlación significativa entre el enfoque estratégico y la puntuación en exámenes teóricos $(r=0,249 ; p=0,004)$.

El análisis también reveló que no hubo correlación del enfoque superficial y el rendimiento académico en cuanto a la puntuación del examen práctico ( $r=$ $-0,027 ; p=0,763)$. No hubo correlación significativa con el enfoque profundo $(r$ 


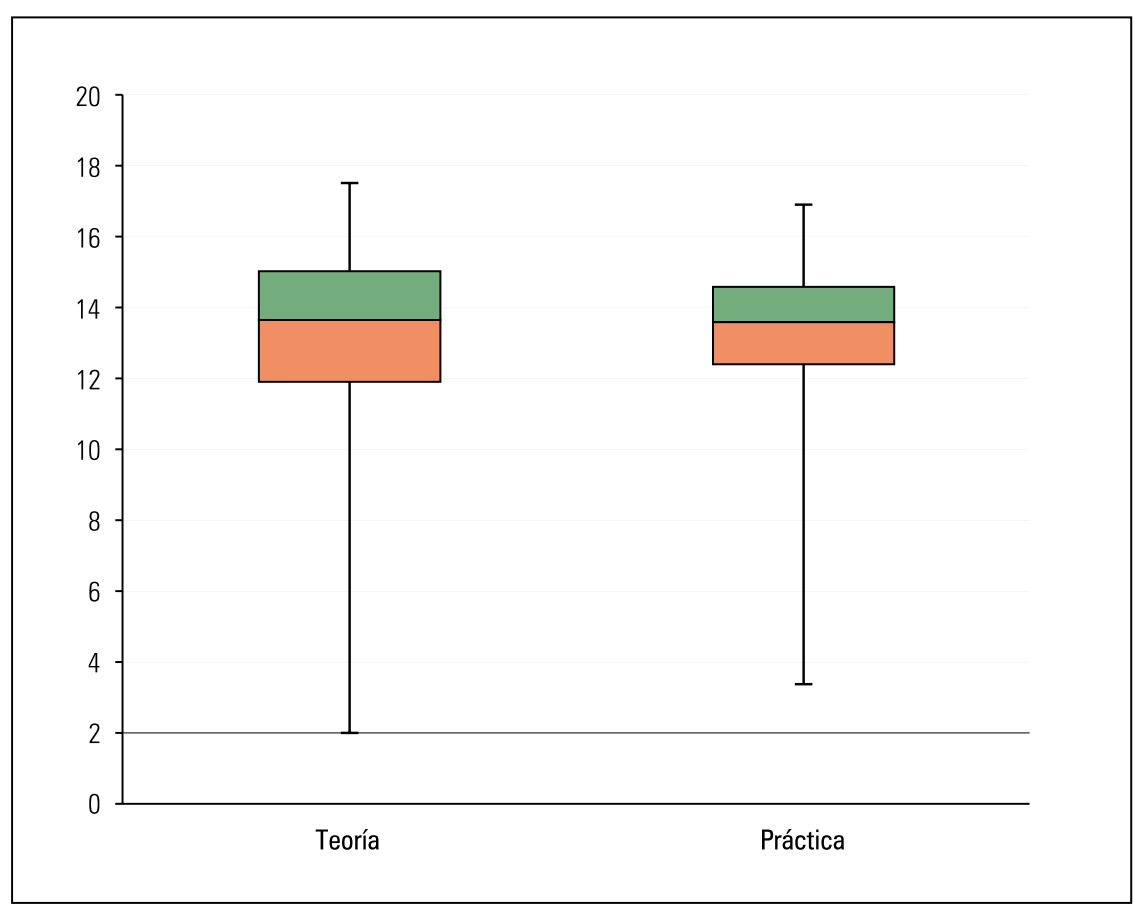

Figura 1. Rendimiento académico en escala vigesimal de las evaluaciones de teoría y de práctica.

$=0,058 ; p=0,515)$. Tampoco hubo correlación significativa entre el enfoque estratégico y la puntuación del examen práctico $(r=0,139 ; p=0,115)$.

Los resultados de la prueba de ANOVA de dos vías revelaron que no hubo un efecto independiente estadísticamente significativo de la edad o el sexo sobre los enfoques de aprendizaje. Del mismo modo, no hubo efecto de la interacción de la edad y el sexo en cada una de las subescalas de los enfoques de aprendizaje. Sin embargo, la interacción entre la edad y el sexo sí resultó estadísticamente significativa para el enfoque superficial. Los valores medios y las desviaciones estándar de cada enfoque de aprendizaje por sexo se presentan en la tabla 4.

\section{DISCUSIÓN}

El análisis de los datos sobre las puntuaciones medias reveló que el enfoque profundo es el preferido de los estudiantes de medicina de primer año, seguido por el enfoque estratégico y el enfoque superficial. Estos resultados concuerdan con los obtenidos en un estudio llevado a cabo en el Reino Unido, donde se halló que los estudiantes de medicina tenían preferencia por el enfoque profundo en comparación con otros estudiantes en la educación superior ${ }^{(6)}$, con resultados similares a otro estudio más reciente en estudiantes de medicina ${ }^{(20)}$. Sin embargo, algunos autores describen las puntuaciones más altas para el enfoque superficial en los estudiantes de medicina bajo el plan de estudios tradicional ${ }^{(21,22)}$. Contrariamente a los hallazgos de la presente investigación y del enfoque deseado para el mejor desempeño de los estudiantes de medicina, el estudio de Newble y Hejkaha ha demostrado que el enfoque profundo no es tan evidente en los estudiantes de medicina (21).

Se encontró que existe una correlación estadísticamente significativa de los tres enfoques. La correlación entre enfoque profundo y estratégico es alta y positiva que significa que estos componentes no son independientes entre sí. En otras palabras, se pueden combinar fácilmente. Por otra parte, la correlación negativa y estadísticamente significativa entre enfoque profundo y superficial significa que estos enfoques son opuestos. Estos hallazgos son concordantes con lo que se espera para este tipo de cuestionario ${ }^{(8,18)}$.

Por otra parte, la correlación entre enfoque superficial y estratégico fue negativa y estadísticamente significativa. Estos hallazgos difieren parcialmente con los obtenidos por Tesouro y colaboradores ${ }^{(20)}$, quienes encontraron una correlación estadísticamente muy significativa y positiva entre enfoque estratégico y enfoque profundo. Sin embargo, en el mismo estudio los resultados de la correlación entre los enfoques profundo y estratégico fueron similares a los reportados en la presente investigación.

Varios informes han demostrado la relación de enfoques de aprendizaje del estudiante y el rendimiento académico. Por ejemplo, en un estudio realizado en estudiantes de psicología, sorprendentemente el enfoque profundo no pudo predecir el rendimiento académico; sin embargo, el rendimiento académico reflejado en las notas de los exámenes estaban relacionados de preferencia por los enfoques superficial y estratégico ${ }^{(7)}$. En nuestro estudio, de manera similar, hubo una correlación significativa entre el enfoque estratégico y calificaciones en el examen teórico. Sin embargo, no se detectó evidencia alguna acerca de la relación entre cada enfoque de aprendizaje y las notas de los exámenes de práctica. No se halló correlación entre las evaluaciones de teoría y el enfoque profundo, ni en la puntuación de los exámenes prácticos y el enfoque profundo.

Algunos estudios relacionan sexo y edad con los enfoques de aprendizaje. Según lo indicado por Ferguson ${ }^{(23)}$, poca investigación se ha hecho sobre la importancia del género y su relación con el rendimiento académico en estudiantes de medicina. Los estudiantes mayores, en comparación con los estudiantes más jóvenes, son más propensos a adoptar el enfoque profundo en su trabajo académico ${ }^{(24)}$. Los estudiantes de medicina varones utilizan preferentemente el enfoque de aprendizaje superficial ${ }^{(6)}$. Sin embargo, algunos estudios mostraron evidencia de que los estudiantes de medicina mujeres tienen un mejor ren- 
dimiento académico y que se relaciona con el enfoque profundo de aprendizaje ${ }^{(25,26)}$. Por otra parte, existen resultados contradictorios en relación a la edad y los enfoques de aprendizaje ${ }^{(26,27)}$. En nuestro estudio no se hallaron diferencias significativas de los enfoques de aprendizaje en relación al sexo y edad.

Se concluye que el enfoque de aprendizaje predominante en los estudiantes de medicina de primer año bajo el actual plan de estudios fue el enfoque profundo, no habiendo diferencias en relación al sexo y edad. Se encontró alta correlación solo entre el enfoque estratégico y los exámenes de teoría. No se hallaron correlaciones con los enfoques de aprendizaje y la puntuación del examen práctico.

Los resultados potenciales que establecen algún tipo de relación entre el enfoque de aprendizaje y los resultados académicos de este estudio no pueden generalizarse en el contexto amplio, sobre todo si la intención es predecir el rendimiento académico al final de los estudios médicos. Sería más conveniente realizar estudios similares con el mismo grupo de estudiantes para seguir los cambios en los enfoques de aprendizaje y rendimiento académico en el tiempo para tener más elementos para una predicción más precisa.

\section{REFERENCIAS BIBLIOGRÁFICAS}

1. Smith SN, Miller RJ. Learning approaches: Examination type, discipline of study, and gender. Educat Psychol. 2005;25(1):43-53.

2. Crosby RH, Joy. AMEE Guide No 20: The good teacher is more than a lecturer-the twelve roles of the teacher. Medical Teacher. 2000;22(4):334-47.

3. Spencer JA, Jordan RK. Learner centred approaches in medical education. BMJ. 1999;318(7193):1280-3.

4. Byrne M, Flood B, Willis P. The relationship between learning approaches and learning outcomes: a study of Irish accounting students. Accounting Educat. 2002;11(1):27-42.

5. Reid W, Duvall E, Evans P. Can we influence medical students' approaches to learning? Medical Teacher. 2005;27(5):401-7.

6. Mattick K, Dennis I, Bligh J. Approaches to learning and studying in medical students: validation of a revised inventory and its relation to student characteristics and performance. Medical Educat. 2004;38(5):535-43.

7. Diseth $\AA$, Martinsen $\varnothing$. Approaches to learning, cognitive style, and motives as predictors of academic achievement. Educat Psychol. 2003;23(2):195-207.

8. Diseth $\AA$. Validation of a Norwegian version of the Approaches and Study Skills Inventory for Students (ASSIST): application of structural equation modelling. Scandin J Educat Res. 2001;45(4):381-94.

9. Abraham RR, Vinod P, Kamath M, Asha K, Ramnarayan $\mathrm{K}$. Learning approaches of undergraduate medical students to physiology in a non-PBL-and partially PBL-oriented curriculum. Advances Physiol Educat. 2008;32(1):35-7.

10. Cope C, Staehr L. Improving students' learning approaches through intervention in an information systems learning environment. Studies in Higher Education. 2005;30(2):181-97.

11. Ellis RA, Goodyear P, Calvo RA, Prosser M. Engineering students' conceptions of and approaches to learning through discussions in face-to-face and online contexts. Learning and Instruction. 2008;18(3):267-82.

12. Lievens F, Coetsier P, De Fruyt F, De Maeseneer J. Medical students' personality characteristics and academic performance: a five-factor model perspective. Medical Educat. 2002;36(11):1050-6.

13. Samarakoon L, Fernando T, Rodrigo C, Rajapakse S. Learning styles and approaches to learning among medical undergraduates and postgraduates. BMC medical education. 2013;13(1):42.

14. Entwistle N, editor Promoting deep learning through teaching and assessment: conceptual frameworks and educational contexts. TLRP conference, Leicester; 2000.
15. May W, Chung E-K, Elliott D, Fisher D. The relationship between medical students' learning approaches and performance on a summative high-stakes clinical performance examination. Medical Teacher. 2012;34(4):e236-e41.

16. UNMSM FdM. Plan de Estudios de la Escuela Académico Profesional de Medicina Humana [05 Mayo 2016]. Disponible en: http://medicina.unmsm.edu. pe/index.php/medicina/plan-de-estudios.

17. Boyle EA, Duffy T, Dunleavy K. Learning styles and academic outcome: The validity and utility of Vermunt's Inventory of Learning Styles in a British higher education setting. Brit J Educat Psychol. 2003;73(2):267-90.

18. Entwistle N. The approaches and study skills inventory for students (ASSIST). Edinburgh: Centre for Research on Learning and Instruction, University of Edinburgh. 1997.

19. Gliem JA, Gliem RR, editors. Calculating, interpreting, and reporting Cronbach's alpha reliability coefficient for Likert-type scales2003: Midwest Research-to-Practice Conference in Adult, Continuing, and Community Education.

20. Tesouro i Cid M, Cañabate Ortiz D, Puiggali J. Los enfoques de aprendizaje en estudiantes universitarios Catalanes mediante el approaches and study skills inventory for students (ASSIST) $=$ Learning Approaches of Catalan University Students Measured with the Approaches and Study Skills Inventory for Students (ASSIST). Revista de Investigacion Educativa, 2014;32(2):479-98.

21. Newble DI, Hejka EJ. Approaches to learning of medical students and practising physicians: some empirical evidence and its implications for medical education. Educat Psychol. 1991;11(3-4):333-42.

22. Newble D, Entwistle N. Learning styles and approaches: implications for medical education. Medical Educat. 1986;20(3):162-75.

23. Ferguson $E$, James $D$, Madeley L. Factors associated with success in medical school: systematic review of the literature. BMJ. 2002;324(7343):952-7.

24. Richardson JT. Mature students in higher education: II. An investigation of approaches to studying and academic performance. Studies in Higher Education. 1995;20(1):5-17.

25. Papinczak T, Young L, Groves M, Haynes M Effects of a metacognitive intervention on students approaches to learning and self-efficacy in a first year medical course. Advances Health Sci Educat. 2008;13(2):213-32.

26. Wilson I, Weaver R, Salamonson Y. Changes in learning approaches in first-year medical students. Focus on Health Professional Education: A Multidisciplinary J. 2011;13(2):65.

27. Andreou E, Vlachos F, Andreou G. Approaches to studying among Greek university students: the impact of gender, age, academic discipline and handedness. Educat Res. 2006;48(3):301-11. 